\title{
A Method to Improve the Demodulation Speed of Digital Signal
}

\author{
He Jing, Liu Yang
}

China Satellite Maritime Tracking and Control Department, Jiangyin, 214431, China

Keywords: PCM-DQPSK; demodulation speed; optimization

\begin{abstract}
In this paper, the DSTFT algorithm is optimized from the point of view of improving system demodulation speed. For the sliding windowing process, this paper gives two fast implementation schemes of DSTFT - recursive and code-sliding. The recursive algorithm of rectangular window and all - pole window is introduced in detail, and their demodulation performance is analyzed. The demodulation idea of DSTFT code - free sliding algorithm is given, and its feasibility is proved theoretically.
\end{abstract}

\section{Introduction}

For demodulation, the validity is the speed of demodulation. The DQPS-based DQPSK signal demodulation system relies on a core algorithm for software programming to implement, but the computational complexity of the algorithm is large. Therefore, this paper optimizes the algorithm from the angle of improving the demodulation speed, and designs the fast implementation scheme of DSTFT. This paper introduces two recursive algorithms for window functions - rectangular window recursion and full pole window recursion.

\section{A recursive algorithm using a rectangular window}

The length of the rectangular window is $M$, the number of points of the DFT operation is also M, the spectral line corresponding to the center frequency is $\mathrm{kc}$, and the $\mathrm{M}$ sampling data from the first sampling point is denoted by $X_{1}\left(k_{c}\right)$ for the DFT operation at the center frequency position Of the Fourier value.

$$
X_{1}\left(k_{c}\right)=x(0)+x(1) e^{-j 2 \pi k_{c} / M}+\cdots+x(M-1) e^{-j 2(M-1) \pi k_{c} / M}
$$

The rectangular window slides back to a sampling point to obtain the Fourier value corresponding to the second sample point:

$$
X_{2}\left(k_{c}\right)=x(1)+x(2) e^{-j 2 \pi k_{c} / M}+\cdots+x(M) e^{-j 2 M \pi k_{c} / M}
$$

The only difference between the formula (1) and the formula (2) is the difference between the data sets, and the exponential terms of the two types are exactly the same. It is easy to see that the formula (2) can be obtained by simply modifying the formula (1), which can be written as:

$$
X_{2}\left(k_{c}\right)=\left[X_{1}\left(k_{c}\right)-x(0)\right] e^{j 2 \pi k_{c} / M}+x(M) e^{-j 2 \pi k_{c}}
$$

The general expression of the above equation can be written as:

$$
X_{n+1}\left(k_{c}\right)=\left[X_{n}\left(k_{c}\right)-x(n)\right] e^{j 2 \pi k_{c} / M}+x(n+M) e^{-j 2 \pi k_{c}}
$$

Thus, the Fourier number (complex number) of the entire sampled data is obtained by the recursive operation of the equation (4), and the phase sequence can be obtained by finding the phase. The use of rectangular window recursive algorithm demodulation, but will slide the DFT process with a recursive operation method, will not cause the loss of demodulation performance.

The error of the DQPSK signal is simulated by using the rectangular window recursive algorithm. The error of the statistical instantaneous phase estimation is shown in Fig. 1, the code rate of the DQOSK signal is $1.563 \mathrm{Mbps}$, the carrier frequency is $2.1 \mathrm{MHz}$, the sampling rate is $16 \mathrm{Msps}$, Is 12.8 $\mathrm{dB}$, and the window length of the rectangular window is 8 . 


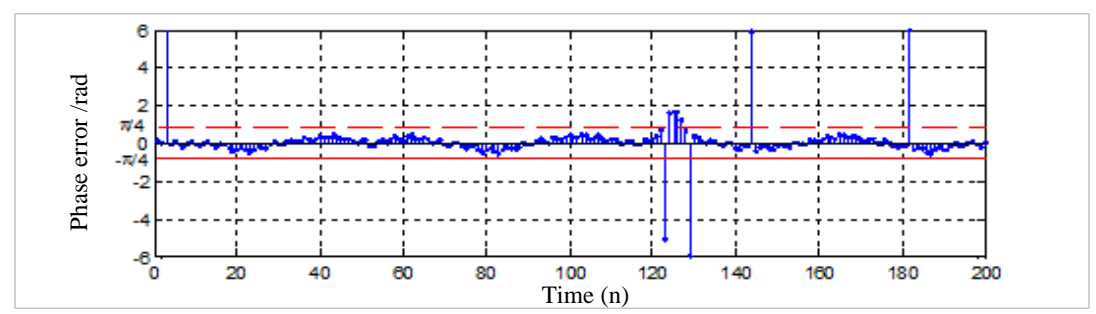

Figure 1. Rectangular window recursive instantaneous phase error

As can be seen from Figure 1, the use of rectangular window recursion does not affect the instantaneous phase of the estimation accuracy, still able to meet the DSTFT demodulation requirements.

\section{A recursive algorithm using the full pole window}

The recursive algorithm of rectangular window makes the demodulation speed increase obviously, but the error rate of rectangular window as demodulation is poor. In order to balance the bit error rate performance of the system, another window can be recursively calculated Function - full pole window.

P-order all-pole window function Z-domain expression is:

$$
W(z)=\frac{1}{\left(1-\beta z^{-1}\right)^{p}}, \quad 0<\beta<1, p=2,3, \cdots
$$

Since W (z) has only a pole, it is called a full pole window. The appropriate order of W (z) is chosen so that the time window w (n) has a single peak characteristic and a desired effective width.

Let the discrete time signal be $\mathrm{x}(\mathrm{n})$, then:

$$
S_{x}(M, \omega)=\sum_{n=0}^{M} x(n) w(M-n) e^{-j \omega n}
$$

After a series of deductions, assuming that the total number of pits is equal to 3, then the type can be written as:

$$
\tilde{S}_{x}(z, \omega)=X(z)+3 \beta e^{-j \omega} z^{-1} \tilde{S}_{x}(z, \omega)-3 \beta^{2} e^{-j 2 \omega} z^{-2} \tilde{S}_{x}(z, \omega)+\beta^{3} e^{-j 3 \omega} z^{-3} \tilde{S}_{x}(z, \omega)
$$

In the time domain, the formula (7) becomes a recursive equation:

$$
\tilde{S}_{x}(n, \omega)=x(n)+3 \beta e^{-j \omega} \tilde{S}_{x}(n-1, \omega)-3 \beta^{2} e^{-j 2 \omega} \tilde{S}_{x}(n-2, \omega)+\beta^{3} e^{-j 3 \omega} \tilde{S}_{x}(n-3, \omega)
$$

The formula (8) is delayed by M sampling points:

$$
S_{x}(n, \omega)=\tilde{S}_{x}(n-M, \omega)
$$

The software demodulation using the short-time Fourier transform of the third-order all-pole window only needs to calculate the Fourier transform value of the first three points of the signal according to the basic formula, and then it can be deduced according to the formula (8) and the formula (9) The Fourier of the entire signal, without the need for DFT operations.

The time-domain expression of the third-order all-pole window function is:

$$
\omega_{3}(n)=\frac{1}{2}(n+1)(n+2) \beta^{n}, \quad n=0,1,2, \cdots
$$

Window length of 100, the location of different peaks of the third-order all-pole sliding window time-domain waveform shown in Figure 2.

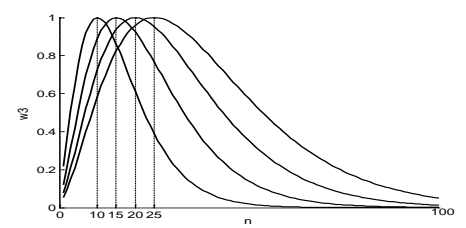

Figure 2. Full - point sliding window time - domain waveform

The window function w3 (n) has the characteristics of a single peak, asymmetry and no sidelobe in the frequency domain, and has the following relation: 


$$
\beta=\exp \left(-\frac{3+2 l_{p}}{2+3 l_{p}+l_{p}^{2}}\right)
$$

For the different order of the all-pole window function, the higher the order, the better the demodulation performance, but the greater the calculation. Therefore, the choice of order should be based on the actual needs of the project to consider, this article selects the third-order all-pole window. The process of demodulating the DQPSK signal using the all-pole window recursive algorithm is simulated. The error of the statistical instantaneous phase estimation is shown in Fig.

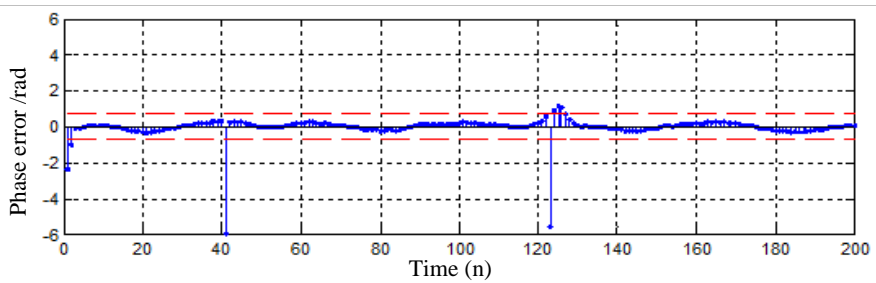

Figure 3. Full - pole window recursive instantaneous phase error

Compared with Fig. 1 and Fig. 3, it can be seen that the precision of the all-pole window is much higher than that of the rectangular window. Therefore, the recursive algorithm using the all-pole window can satisfy the system's requirement of demodulation speed and the system Bit rate performance requirements, is an ideal analysis window function.

\section{DSTFT Slip-Slip Realization}

Whether it is the basic DSTFT demodulation or the use of its recursive algorithm, the window function is always sliding by the sampling point, and the instantaneous phase sequence of the whole signal length is estimated. The difference decision only uses the instantaneous phase value at the best decision point, and the calculated majority of the sampling point of the phase has not been used, resulting in a great waste. Therefore, the design of DSTFT another implementation program DSTFT code-free sliding algorithm, the main idea is: first to achieve bit synchronization, find the best decision point, and then only estimate the best decision point instantaneous phase and the difference judgment.

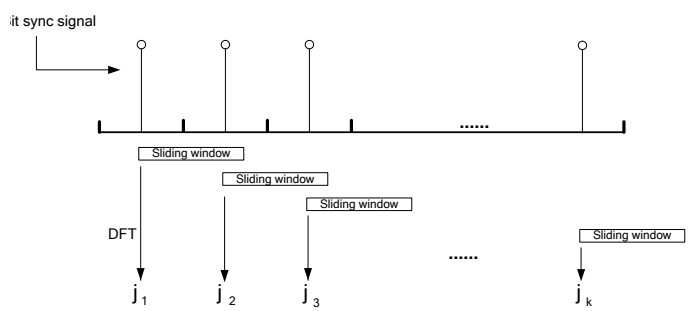

Figure 4. Slide the DSTFT phase diagram by code

It can be seen from Fig. 4 that the DSTFT of each double bit code only needs to calculate the instantaneous phase at a sampling point, and the computation is reduced to $R_{b} / 2 f$ of the original. For the parameters of this paper, the amount of computation becomes the original $1 / 21$ or so, which is to improve the system processing speed is undoubtedly a significant effect.

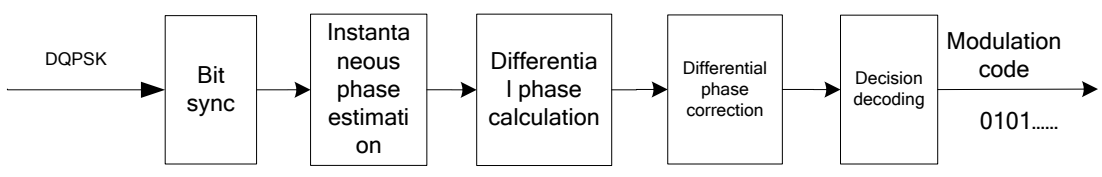

Figure 5. DSTFT block diagram of code - free sliding algorithm

Figure 5 is a block diagram of the system using DSTFT's code-free sliding algorithm. First start bit synchronization positioning symbol of the starting position, and then find the best decision point. Then use the DSTFT algorithm to estimate the instantaneous phase at the optimal decision point, calculate the difference phase and correct it. Judgment decoding directly on the front of the instantaneous phase of the decision, the phase mapping output binary stream. It should be noted that, when performing differential phase correction, can not be calculated according to the formula, 
because the bit synchronization is not ideal, the best decision between the code between the number of points is no longer the theoretical value, no longer equal The So the bit synchronization need to set the counter, record the number of sampling points between each decision points, to correct the inherent phase difference between the symbols.

Because DSTFT's code-less sliding algorithm only omit most of the sampling point of the DFT operation, does not affect the processing results, so if the bit synchronization is correct, the demodulation performance will not be any deterioration. In addition, because the amount of computing has been greatly reduced, do not have to use a limited number of recursive algorithms, and can choose the best window function, so even to a certain extent, improve the system's bit error rate performance.

DSTFT code-based sliding algorithm to achieve the median synchronization is the key, directly determine the correctness of the demodulation results, on the bit synchronization algorithm research and discussion see Chapter IV.

\section{Summary}

This paper optimizes the DSTFT algorithm from the point of view of improving system demodulation speed. For the sliding windowing process, this paper gives two fast implementation schemes of DSTFT - recursive and code-sliding. The recursive algorithms of rectangular windows and all - pole windows are introduced in detail, and their demodulation performance is analyzed. The feasibility of DSTFT code - sliding algorithm is theoretically demonstrated.

\section{Reference:}

[1] L.Erup, F,M,Gardner, interpolation in digital modems-part II. Implementation and performance, IEEE. Trans. Com, Jun.2013, 41(6):998-1008.

[2] Chuang J.C, Sollenberger N.R, Burst coherent demodulation with combined symbol timing frequency offset estimation, and diversity selection. IEEE,Trans.Com, 2011, 39(7):1157-1164.

[3] Sollemberger N.R, Chuang J.C, Low Overhead symbol timing and carrier recovery for TDMA portable radia systems. IEE, Trans.Com, 2010, 38(10):1886-1892.

[4] G.Ascheid, M.oerder, H.meyr, An All Digital Receiver Architecture for Bandwidth Efficient Transmussion at high Rates, IEEE, Trans. Com, Aug.2009, 37(8):804-813. 